\title{
Research Article \\ Effect of Polymer Adsorption on Permeability Reduction in Enhanced Oil Recovery
}

\author{
Saurabh Mishra, Achinta Bera, and Ajay Mandal \\ Department of Petroleum Engineering, Indian School of Mines, Dhanbad 826004, India \\ Correspondence should be addressed to Ajay Mandal; mandal_ajay@hotmail.com
}

Received 25 November 2013; Revised 24 January 2014; Accepted 27 January 2014; Published 5 March 2014

Academic Editor: Alireza Bahadori

Copyright (C) 2014 Saurabh Mishra et al. This is an open access article distributed under the Creative Commons Attribution License, which permits unrestricted use, distribution, and reproduction in any medium, provided the original work is properly cited.

\begin{abstract}
In order to reduce the permeability to water or brine, there is a possibility of polymer injection into the reservoir. In the present work, special focus has been paid in polymer [partially hydrolyzed polyacrylamide (PHPA)] injection as a part of chemical method. Tests were conducted in the laboratory at the ambient temperature to examine the reduction in permeability to water or brine in the well-prepared sand packed after the polymer injection. The experiments were performed to study the effect of polymer adsorption on permeability reduction by analyzing residual resistance factor values with different concentrations of polymer solutions. The rheological behavior of the polymer has also been examined. The experimental results also indicate that the adsorption behavior of polymer is strongly affected by salinity, solution $\mathrm{pH}$, and polymer concentration. To investigate the effect of polymer adsorption and mobility control on additional oil recovery, polymer flooding experiments were conducted with different polymer concentrations. It has been obtained that with the increase in polymer concentrations, oil recovery increases.
\end{abstract}

\section{Introduction}

With reservoir getting matured, the increased water production with producing oil is a major concern in the petroleum industry. Hydrocarbon production decreases, which affects recovery economics and disposal of the excessive high amount of underground water, which causes complex environmental problems [1]. Oil and gas reservoirs are often heterogeneous, having a different permeability in differentdifferent layers. This causes channelling of excessive water production through high permeability layers, as a result of which large amount of movable oil and gas remains trapped in low permeability zones which results in poor recovery in primary and secondary stages of production. A significant part of the residual oil can be recovered by application of a polymer solution in the heterogeneous reservoirs [2]. The excess water production can be controlled without affecting oil production rate by using polymer to reduce the relative permeability to water more than the relative permeability to oil [3]. The injection of polymer solutions in production wells has proven to be an effective method to reduce or block excessive water production [4]. In a water-wet reservoir, oil flows inside pores while water through the annulus between the pore walls and the oil-water interface [5]. When the polymer solution is injected, it gets adsorbed on the rock surface to form a thin layer. As water flows through this zone, the adsorbed polymer swells and prevents the flow of water while allowing the oil to flow through. When the polymer is injected, it builds up a resistance to flow in the reservoir through permeability reduction. This increased flow resistance diverts the injected water into unswept areas [6]. Interestingly, the adsorbed polymer is sustained over a long time; this leads to a great reduction in permeability [7]. In 1974, some of the basic concerns pertaining polymer adsorption in porous medium and effect of polymer adsorption on flow properties have been investigated by Hirasaki and Pope [8]. They reported that polymer treatment reduces permeability by adsorption of polymer and reduction in pore radii. The permeability reduction caused by polymer adsorption was traditionally known as "residual resistance factor" which is tantamount to the endpoint relative permeability [9].

Laboratory experiments have been performed by many researchers to examine polymer retention and its effect 
on oil recovery. Zaitoun and Bertin showed in their work that oil and water permeability can be modified by wall effects due to adsorption of polymer [10]. Grattoni et al. reported atomic force microscopy study on polymer retention on microglass surface. They discussed the basic adsorption mechanism and flow of polyacrylamide in the formation of layer thickness [11]. Lai et al. have recently showed the permeability reduction performance of a hyperbranched polymer in high permeability porous media [12]. Al-Hashmi and Luckham studied the effect of salt concentration on the adsorption of high-molecular-weight nonionic and cationic polymers on glass surface and showed that with increase in salt concentration the adsorbed layer thickness also increases [13]. Al-Sharji et al. reported that polymer adsorption significantly influences the water permeability and enhances the oil recovery [14].

In polymer flooding technique, a high-molecular-weight water-soluble polymer is added as a thickening agent into injected water to increase viscosity of aqueous phase for mobility control which can improve sweep efficiency. One of the attractive properties of such polymers is their ability to reduce the relative permeability to water more than the relative permeability to oil in porous media. Liu et al. reported in their framework in the Daqing oil field that from polymer flooding water cut was significantly reduced and oil recovery was improved by $10 \%$ [15]. Polymer flooding projects yielded additional recoveries of $10-15 \%$ of the original oil in place. Since the efficiency of polymer flooding differs with the rock and fluid properties as it varies from field to field. Thus an efficient work is required for improvement of this technology in practical field [16].

In this work, Partially Hydrolyzed Polyacrylamide (PHPA) is used to examine the effect of polymer adsorption on the permeability modification and oil recovery in a sand pack system. Rheological property of the used polymer has also been investigated by measuring the viscosity of different concentrated polymer solutions with a variation of shear rate. Adsorption experiments have been carried out with a variation of polymer concentrations, salinity, and $\mathrm{pH}$ of the solution. Along with this, thickness of the adsorbed layer of polymer has also been calculated with the help of the permeability reduction data. Flooding experiments have been performed to investigate the additional oil recovery after conventional water flooding.

\section{Experimental Sections}

2.1. Materials Used. Partially hydrolyzed Polyacrylamide (PHPA) (Polymer Pusher 1000, SNF Floerger, France) with 98\% purity was supplied by Central Drug House (CDH) Pvt. Ltd., India. Typical degree of hydrolysis of the used polymer is $30 \%$ to $35 \%$. Sodium Chloride $(\mathrm{NaCl})$ with $98 \%$ purity, procured from Qualigens Fine Chemicals, India, was used for preparation of brine. Reverse osmosis water from Millipore water system (Millipore SA, 67120 machine, France) was used for the preparation of solutions. Sand particles (60-70 mesh sized) were used as adsorbent in the adsorption study. The details compositional analysis of the used sand particles has been given in our previous work [17]. The crude oil used in the flooding experiments was collected from the Ahmedabad oil field (India). It was degassed and dehydrated before use. The physical properties of crude oil are shown as follows:

(i) viscosity: $5.12 \mathrm{~Pa} \cdot \mathrm{s}$ at $45^{\circ} \mathrm{C}$ at $20 \mathrm{~s}^{-1}$ shear rate,

(ii) total acid number: $0.038 \mathrm{mg} \mathrm{KOH} / \mathrm{g}$,

(iii) API gravity: 38.5,

(iv) color: blackish.

\subsection{Experimental Procedures}

2.2.1. Preparation and Rheology of Polymer Solution. Polymer solutions were prepared by dissolving the required amount of polymer in brine. Polymer solutions were prepared by the slow addition of the polymer powder to the brine in a vortex created by a magnetic stirrer. Gentle agitation was maintained over day for the complete polymer dissolution to obtain a homogeneous solution. Care was taken to rotate the magnetic stirrer at low rpm speed to avoid any mechanical degradation. In the present study, the rheological property of the used polymer was measured with the help of a rheometer (Physica MC1) at different concentrations. The flow curves were recorded between $10^{-1}$ and $10^{3} 1 / \mathrm{s}$ shear rate using a linear rate program and $2 \mathrm{~min}$ ramp time.

2.2.2. Permeability Reduction by Polymer Flooding. Sand packed was mounted horizontally in a sand packed holder and flow experiment was performed. First sand packed was flooded with brine and initial permeability to brine was calculated. Polymer solution was then injected, when sand packed was fully saturated with brine. The flow rate was measured during polymer flooding. When the flow rate attained a constant value, the polymer injection was stopped. The sand packed was then kept at rest for 50-56 hours for maximum interaction between sand particles and polymer molecules. Brine was then injected at constant pressure and flow rate curve was drawn. Above procedure was repeated for each concentration of polymer solution.

\subsubsection{Polymer Adsorption and Thickness of Adsorbed Layers.} A series of batch experiments were carried out to determine the adsorption of PHPA on the sand particles. $100 \mathrm{~g}$ of clean sand particles were added to $100 \mathrm{~mL}$ polymer solution in a $250 \mathrm{~mL}$ conical flux and allowed to conduct the experiment by constant shaking at $303 \mathrm{~K}$ for $24 \mathrm{~h}$ on a horizontal shaker mode. Core flooding is a dynamic process. So the dynamic condition was considered to maintain the polymer flooding process. During the polymer injection pressure was applied therefore the dynamic condition is maintained. Actually dynamic condition is an appropriate field relevant condition. It is important to mention that the ratio of adsorbent to adsorbate also varies for different studies [18, 19]. In the present study, as flooding experiment was conducted on sand packed, the ratio was taken in this pattern. Again a certain amount of adsorbent is required for maximum adsorption. So a large excess concentration of adsorbent was 
taken in this study. After adsorption, the polymer solutions were isolated by centrifugation. The equilibrium or residual concentrations of the polymer solutions were determined by UV-spectrophotometer. The equilibrium concentrations $\left(C_{e}\right)$ were calculated by using Beer-Lambert equation:

$$
A=\varepsilon * L * C,
$$

where $A$ is absorbent, $\varepsilon$ is molar extinction coefficient $(\mathrm{L} / \mathrm{mole} / \mathrm{cm}), L$ is the path length $(10 \mathrm{~mm})$, and $C$ is concentration $(\mathrm{mole} / \mathrm{L})$ of the solution.

The adsorption capacity of polymer on the adsorbent, $\Gamma_{i}$ $(\mathrm{mg} / \mathrm{g})$, was calculated by a mass balance relation [20]:

$$
\Gamma_{i}=\left(C_{0}-C_{e}\right) \frac{V}{m},
$$

where $C_{0}$ and $C_{e}$ are the initial and equilibrium concentrations of polymer solutions $(\mathrm{mg} / \mathrm{g})$, respectively, $V$ is the volume of the polymer solution (L), and $m$ is the weight of the sand particles (g) (adsorbent) used. The effect of salt concentration, $\mathrm{pH}$, and polymer concentrations is investigated. These may greatly affect polymer adsorption hence permeability reduction and polymer flood efficiency.

The thickness of the adsorbed layer of polymer $\left(\chi_{p}\right)$ can be calculated from the permeability reduction data. The calculation is based on the assumption of Poiseuille's fluid flow through a capillary having its cross-section reduced by a uniform layer. The thickness of the adsorbed layer of polymer is calculated as follows [3]:

$$
\chi_{p}=r_{p}\left(1-\mathrm{RRF}^{-1 / 4}\right) \text {, }
$$

where RRF is the residual resistance factor and $r_{p}$ is the average pore diameter, which can be calculated as [3]

$$
r_{p}=1.15\left(\frac{8 k_{\mathrm{w}}}{\varphi}\right)^{1 / 2},
$$

where $k_{\mathrm{w}}$ is the effective permeability to water and $\varphi$ porosity of the sand pack.

\subsubsection{Experimental Apparatus and Method for Polymer Flood-} ing. The experimental apparatus is composed of a sand pack holder, cylinders for holding chemical slugs and crude oil, positive displacement pump, and measuring cylinders for collecting the samples. The detail of the apparatus and method of flooding is reported in our earlier paper [21]. The schematic diagram of flooding experiment is given in Figure 1. The model geometry size is $L=35 \mathrm{~cm}$ and $r=$ $3.5 \mathrm{~cm}$. The sand packed holder was tightly packed with uniform sands (60-100 mesh) and saturated with $1.0 \mathrm{wt} \%$ brine. The additional recoveries were calculated by material balance.

Darcy's law was used to calculate the effective permeability to oil $\left(k_{\mathrm{o}}\right)$ and effective permeability to water $\left(k_{\mathrm{w}}\right)$ at irreducible water saturation $\left(S_{\mathrm{wi}}\right)$ and residual oil saturation $\left(S_{\text {or }}\right)$, respectively. For a horizontal linear system, flow rate is related to permeability as follows:

$$
q=\frac{k A}{\mu} \frac{d p}{d x}
$$

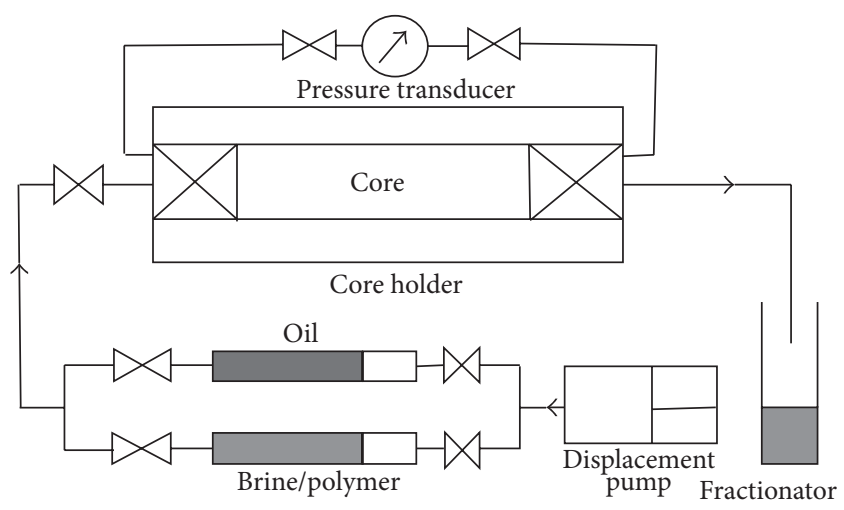

FIGURE 1: Schematic diagram of flooding experiment.

where $q$ is the volumetric flow rate $\left(\mathrm{cm}^{3} / \mathrm{Sec}\right.$.), $A$ is the total cross-sectional area of the sand pack $\left(\mathrm{cm}^{2}\right), \mu$ is the fluid viscosity (cp), $d p / d x$ is the pressure gradient ( $\mathrm{atm} / \mathrm{cm}$ ), and $k$ is permeability in Darcy. Experiments were performed using $2500 \mathrm{ppm}$ and $2000 \mathrm{ppm}$ PHPA solutions.

\section{Results and Discussion}

3.1. Rheology of PHPA Solutions. Polymer rheology plays an important role in controlling the mobility ratio and hence the sweep efficiencies. The basic rheological behavior of aqueous solutions of partially hydrolyzed polyacrylamide has been investigated by varying polymer concentrations. Figure 2 shows the variation of apparent viscosity with shear rate. The viscosity of solutions increased with increasing polymer concentrations because of the increasing intermolecular entanglement. The viscosities of all samples decreased with the increasing shear rate, suggesting that the aqueous solution of PHPA exhibits non-Newtonian behavior. This is due to uncoiling and aligning of polymer chains when exposed to shear flow. As the shear rate approaches toward zero the solutions show maximum viscosity. The viscosity is quite significant at moderate shear rate. It has also been found that at higher shear rate the solution approaches towards Newtonian behavior.

3.2. Permeability Reduction. The flow behavior of polymer solutions of different concentrations and subsequent brine injection through the sand packs are shown in Figure 3. It may be found that as the cumulative injected volume of polymer solution increases the flow rate gradually decreases. This is because of adsorption of polymer on the sand particle. After a certain pore volume injected there is no further change in flow rate. This indicates that the surfaces of sand particles are now fully saturated with polymer and there is no further adsorption. The system is kept as it is for around 50 hours for maximum interaction between polymer and sand particles. The polymer coats on the rock surfaces with a hydrophilic film. As water passes over the polymer, the film swells, reducing the effective permeability. In presence of oil, the swelling does not occur, thereby improving potential oil recovery [22]. Then the sand packs 


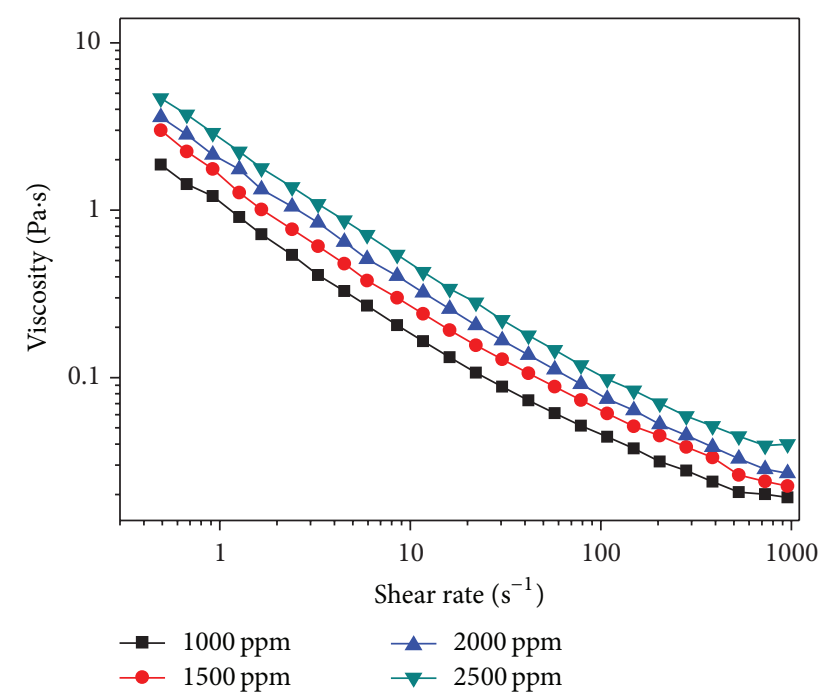

FIGURE 2: Viscosity of PHPA solutions with different concentrations at $303 \mathrm{~K}$.

are continuously injected with brine. Brine flow rate gradually increases due to desorption of polymer. But after a certain pore volume of brine injected it has been found that there is no further increase of flow rate. This suggests that even after a long time of chase water flooding after polymer flooding, a certain amount of polymer is retained and hence the benefits of permeability reduction can be achieved for a long time.

The absolute permeability and the residual permeabilities have been measured by Darcy's law and the results are shown in Figure 4. It has been found from the figure that absolute permeability of the sand pack is much higher than that of residual permeabilities. And the residual permeabilities increase with the increase in concentrations of polymer.

To examine restriction in flow due to polymer adsorption, resistance factor (RF) and residual resistance factor (RRF) ware calculated. Figure 5 shows the values of RF and RRF with a variation of pore volume injected at different polymer concentrations. Initially, when the polymer was injected, it prefers high permeability zones due to low resistance in the flow. Results show that the increment in the polymer concentrations brings to the high value of RF and RRF. Interaction between polymer and sand grains leads to profile modification. This profile modification depends on how polymer is adsorbed on the wall of sand grains in high permeability zones. Due to polymer adsorption pore throat radii reduces, this results in an unexpected decrement in mobility to polymer. The measure of the mobility reduction is known as "resistance factor." Resistance factor was calculated by the following equation:

$$
\mathrm{RF}=\frac{\lambda_{\mathrm{w}}}{\lambda_{\mathrm{p}}}
$$

where $\lambda_{\mathrm{w}}$ is the mobility of water and $\lambda_{\mathrm{p}}$ is the mobility of polymer solution.

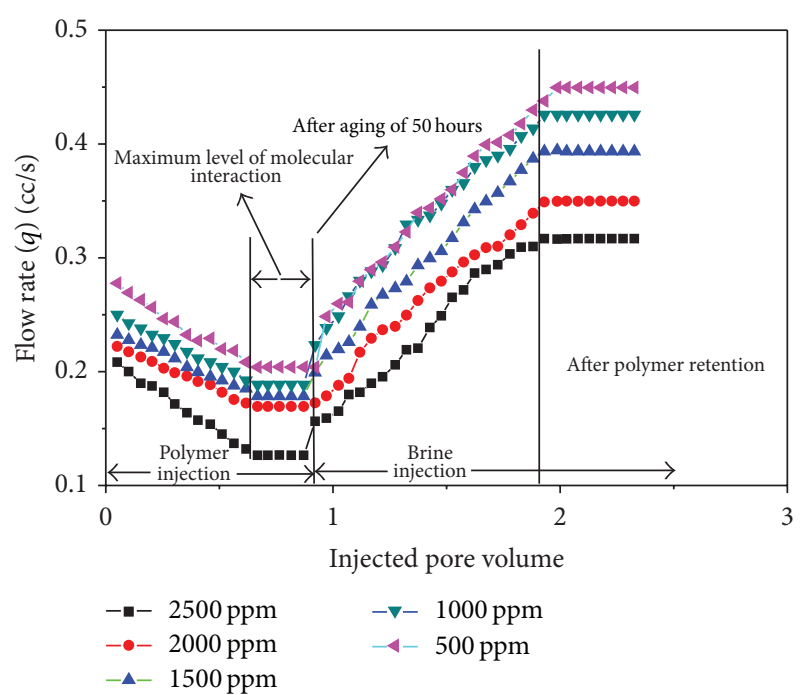

FIGURE 3: Flow rate curves during injection of brine after aging of polymer on sand grains.

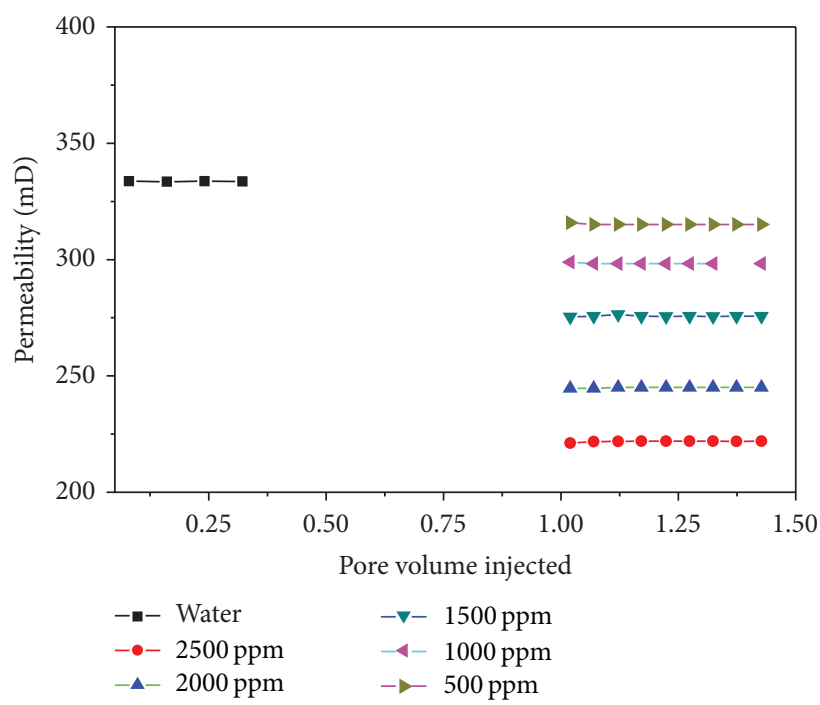

FIGURE 4: Reduction in permeability due to polymer retention.

Mobility is calculated by using Darcy's equation as follows:

$$
\lambda=\frac{q * L}{A * \phi * \Delta p} .
$$

Table 1 shows the calculated data of RF and RRF for PHPA with different concentrations.

3.3. Polymer Adsorption. Adsorption of polymer on rock surfaces is influenced by several factors. The influencing factors are polymer concentrations, salinity of the solution, the $\mathrm{pH}$ of the solution, temperature, and so forth. Some of the factors have been discussed here with experimental results. 
TABLE 1: Calculated values of RF and RRF for different concentrated PHPA solutions.

\begin{tabular}{lccc}
\hline Test samples & Mobility & Resistance factor (RF) & Residual resistance factor (RRF) \\
\hline Water & 1.027 & - & - \\
$C_{1}(2500 \mathrm{ppm}+2 \mathrm{wt} \% \mathrm{NaCl})$ & 0.231 & 4.461 & 1.504 \\
$C_{2}(2000 \mathrm{ppm}+2 \mathrm{wt} \% \mathrm{NaCl})$ & 0.284 & 3.621 & 1.361 \\
$C_{3}(1500 \mathrm{ppm}+2 \mathrm{wt} \% \mathrm{NaCl})$ & 0.327 & 3.143 & 1.211 \\
$C_{4}(1000 \mathrm{ppm}+2 \mathrm{wt} \% \mathrm{NaCl})$ & 0.353 & 2.905 & 1.119 \\
$C_{5}(500 \mathrm{ppm}+2 \mathrm{wt} \% \mathrm{NaCl})$ & 0.369 & 2.783 & 1.059 \\
\hline
\end{tabular}

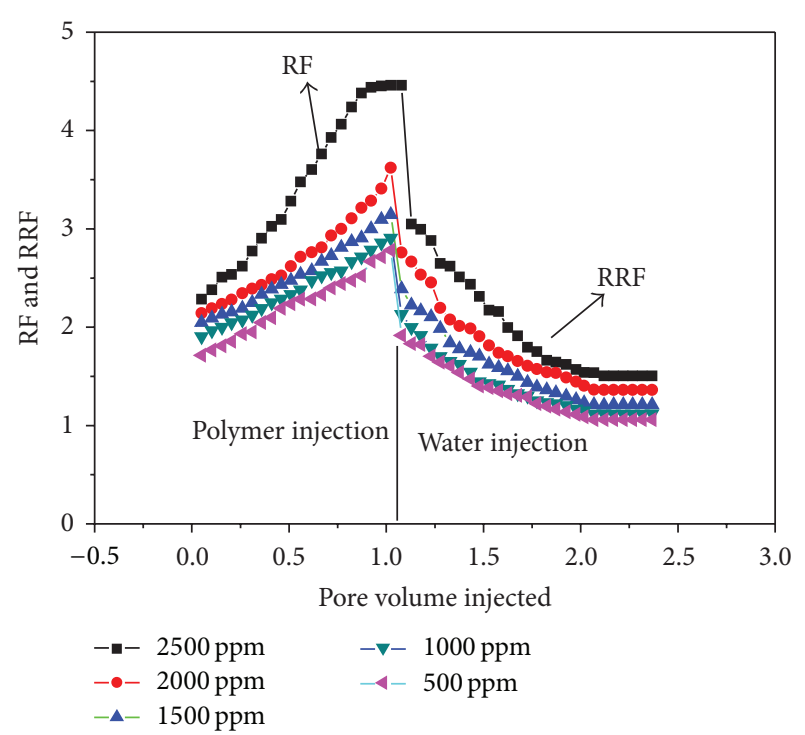

FIGURE 5: Plot of RF and RRF with different concentrations of polymer solution.

3.3.1. Thickness of Adsorbed Layer. Figure 5 shows the relation between polymer concentrations and thickness of adsorbed layer. It is confirmed from Figure 6 that with the increase in polymer concentrations the thickness of adsorbed layer also increases. As concentration increases, the number of molecules increases, thereby the probability of interaction between polymer molecules and sand particles increases. This brought increment in adsorption capacity per unit area. Therefore, it is confirmed that the adsorption layer thickness increases with an increase in adsorption. The increase in the adsorption layer thickness as the polymer content in solution increases is due to a change in the conformation of the adsorbed molecules [23]. The change in the adsorption layer thickness in the initial segments of the isotherms suggests that at low concentrations of polymer, the molecules on the surface of the sand particles are distributed and bound with a small number of segments. The increase in concentrations of polymer causes a rearrangement of the structure of the adsorbed layer. The adsorbed molecules break previously formed bonds so that they straighten and unfold and the layer thickness increases.

3.3.2. Effect of Polymer Concentrations on Adsorption. Figure 7 shows the time dependent polymer adsorption on

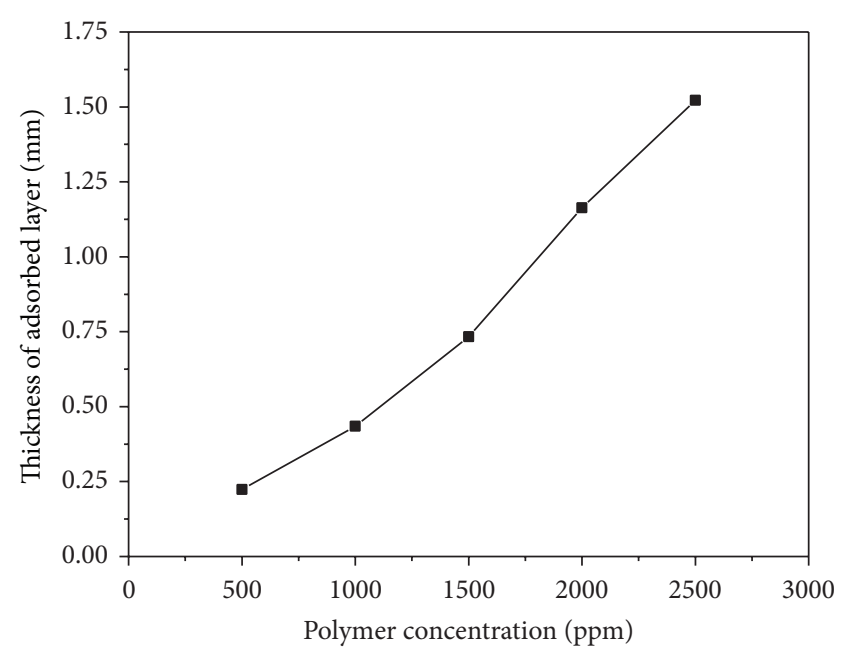

FIGURE 6: Relation between polymer concentrations and thickness of adsorbed layer.

the sand surface at different concentrations. With increase in time polymer adsorption increases and after certain time adsorption remains constant with time. As time increases, the number of adsorption sites decreases due to formation of adsorbed polymer layer on the sand surface. When all the sites are covered with polymer, then further adsorption does not take place and time independent adsorption occurs. Figure 7 shows that with the increase in polymer concentrations the polymer adsorption capacity also increases. At higher concentrations of polymer solutions, number of polymer molecules increases, which in turn increase the probability of interaction between the sand surface and polymer molecule. It is also important to point out here that after certain concentration no further adsorption takes place due to saturation of the adsorption capacity of the active adsorption sites $[9,24,25]$. In the present case, only four different concentrations have been used to show the effect of concentrations on polymer adsorption onto the sand surface and it has been found that up to $2500 \mathrm{ppm}$ concentration of PHPA polymer adsorption increases. Adsorption causes the permeability reduction. Ogunberu and Asghari have reported on their experimental work that increase in polymer concentrations results in an increase in permeability reduction caused by polymer adsorption and efficiency of polymer flooding [3]. 


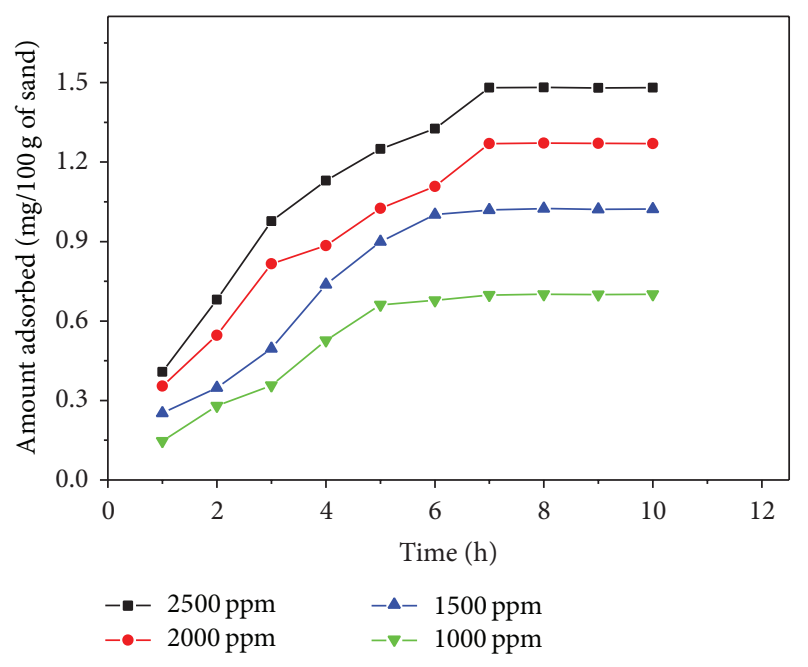

FIGURE 7: Effect of polymer concentrations on polymer adsorption.

3.3.3. Effect of Solution $p H$ on Polymer Adsorption. The adsorption behavior of PHPA at different solution $\mathrm{pH}$ has been shown in Figure 8. The adsorption capacity increases when solution $\mathrm{pH}$ is decreased as shown in Figure 8. At low $\mathrm{pH}$, sand surfaces become more positive due to the acidic nature of the polymer solutions and the interaction of sand surface with anionic polymer PHPA is high, hence, adsorption capacity is high. The negatively charged carboxyl group dissociated through electrostatic attraction to the positively charged ions (due to more $\mathrm{H}^{+}$ions). This causes more attraction between polymer molecules and rock surfaces. When the $\mathrm{pH}$ of the solution increases, that is, the solutions become alkaline in nature, the sand surfaces become more negative. The repulsion between PHPA with negatively charged carboxylic acid group and negatively charged sand surface takes place, which leads to lower adsorption of the polymer on sand surfaces. So, it is confirmed from the study of the effect of $\mathrm{pH}$ on adsorption that the adsorption of polymer on rock surfaces can be modified by fixing the solution $\mathrm{pH}$ which is important for polymer flooding.

3.3.4. Effect of Salt Concentration on Polymer Adsorption. Experimental results have been shown in Figure 9. It is clear from Figure 8 that polymer adsorption increases with increase in salinity. As the salt concentration increases, charge shielding takes place due to positively charged ions of the salt $\left(\mathrm{Na}^{+}\right)$, thus the hydrodynamic radius of polymer molecule reduces. Due to this intermolecular interaction, electrostatic repulsion in the polymer solution decreases. This causes an increase in adsorption capacity of polymer capacity of polymer solution. Moreover the salt concentration may affect the displacement efficiency and volumetric sweep efficiency of the polymer flood. High salt concentration may affect the polymer absorption by an increase in the viscosity of polymer due to electrostatic repulsion in the polymer solution and a reduction in the surface area of adsorbent, access to polymer molecules. Due to the above two parametrical effects, adsorption capacity increases as salt concentration increases.

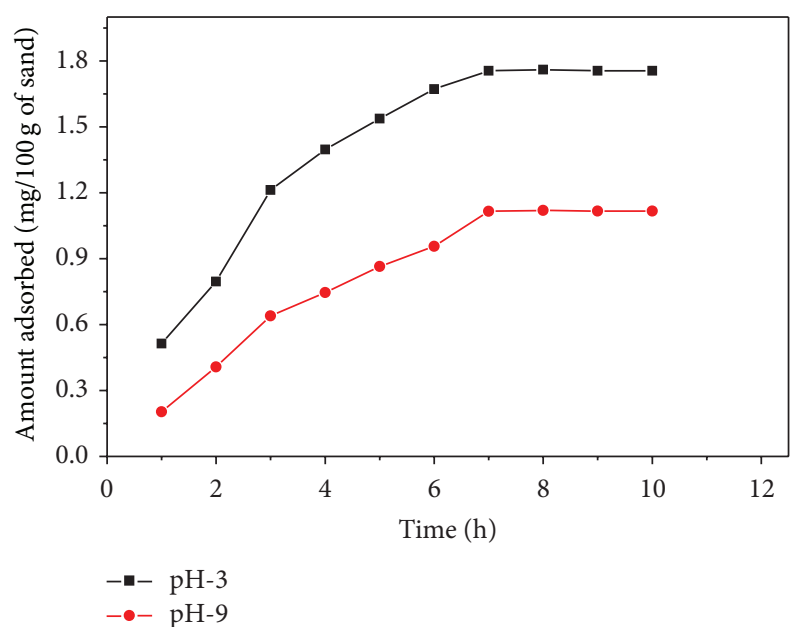

FIGURE 8: Effect of solution $\mathrm{pH}$ on polymer adsorption on sand surface.

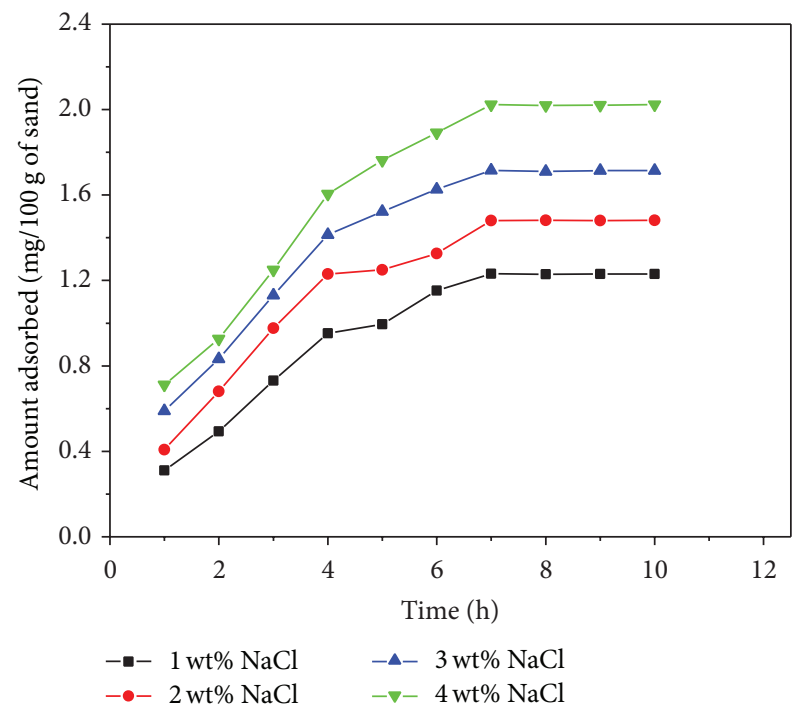

FIGURE 9: Effect of salt concentration on polymer adsorption.

3.4. Oil Recovery by Polymer Flooding. Injection of the polymer increases the recovery of oil by improving the mobility ratio, reducing the effective permeability to water and increasing the effective permeability to water. In the present study, two different concentrations of PHPA were used to measure the additional recovery of oil after conventional water flooding. Initially the sand pack was flooded with brine. When water cut reached around 95\%, approximately $0.75 \mathrm{PV}$ polymer solution was injected to investigate its effect on the efficiency of polymer flooding. Two sets of experiments were performed using $2000 \mathrm{ppm}$ and $2500 \mathrm{ppm}$ polymer solution. The recovery of oil while flooding the sand packed with brine and different concentrations of PHPA are shown in Figure 10. The main parameters and results of these flood tests are in Table 2, including the initial water saturations, the injected pore volumes and oil recoveries during water flooding and 


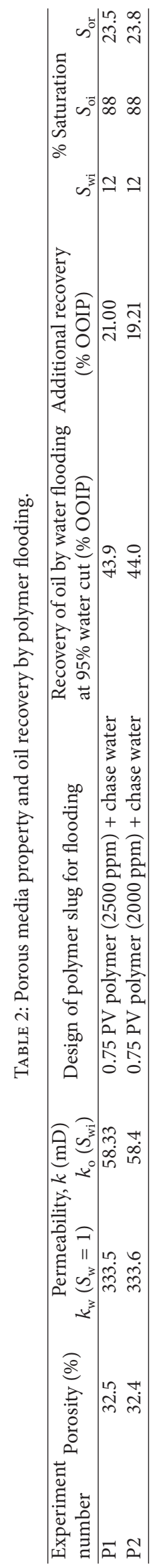




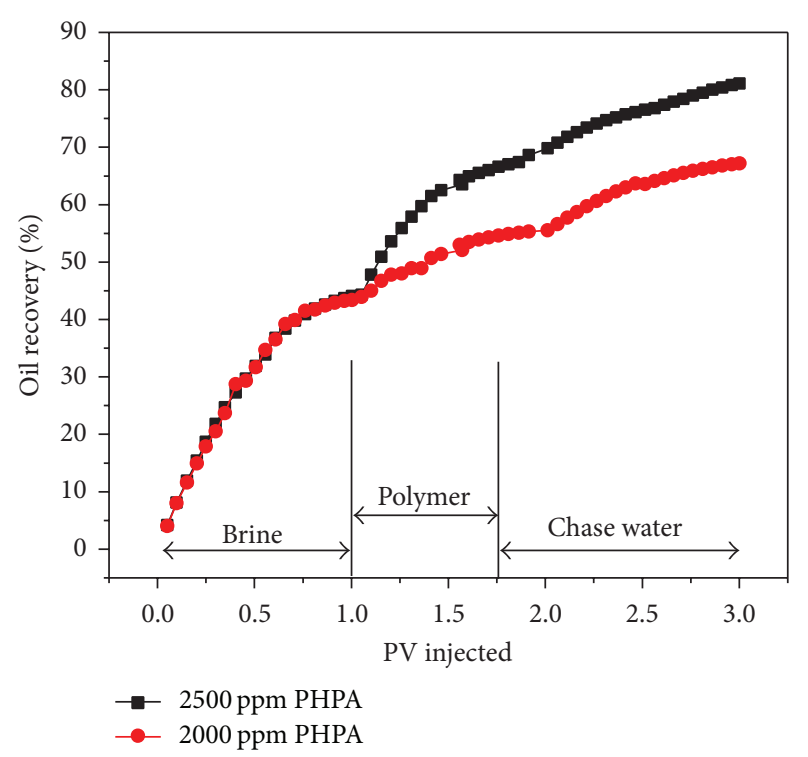

FIGURE 10: Oil recovery by polymer flooding using $2500 \mathrm{ppm}$ and 2000 ppm PHPA solutions.

tertiary recoveries. All recoveries are calculated based on the original oil in place (OOIP).

The additional oil recoveries by polymer flooding were $19.21 \%$ and $21.00 \%$ OOIP for $2000 \mathrm{ppm}$ and $2500 \mathrm{ppm}$ PHPA solutions. These results were compared with another literature reported by Maitin, where the laboratory investigations, simulation studies, and a pilot test in the oil fields of a North German sediment basin have produced the incremental recovery of 8 to $22 \%$ OOIP [26].

\section{Conclusions}

Rheological properties of the PHPA solution have been determined and it has been found that with increase in polymer concentrations viscosity also increases. Adsorption of polymer on sand particles has been studied by polymer flooding through sand packed. Experimental results show that after aging, there was significant interaction between polymer molecules and sand grains, which plugged the flow path to inject brine at constant pressure; as a result, there was a significant decrement in flow rate. As brine injection increased, polymer retention decreased and reached a critical value which gives an absolute permeability reduction. The result shows that increase in polymer concentrations enhances the polymer adsorption capacity. At low $\mathrm{pH}, \mathrm{PHPA}$ adsorption capacity on sand surface is high due to the acidic nature of the solution which makes the sand surface more positive and that is why the interaction of sand surface with anionic polymer PHPA is high, hence, adsorption capacity is high. When the $\mathrm{pH}$ of the solution increases the solution becomes alkaline and as a result repulsion between PHPA and negatively charged sand surface increases, therefore, adsorption of the polymer decreases. As the salt concentration increases, charge shielding takes place due to positively charged ions of the salt $\left(\mathrm{Na}^{+}\right)$, thus the hydrodynamic radius of polymer molecule reduces. Due to this intermolecular interaction, electrostatic repulsion in the polymer solution decreases. This causes an increase in adsorption capacity of polymer capacity of polymer solution. The result shows that with the increase in polymer concentrations, thickness of adsorbed layer also increases. To investigate the relationship between the incremental tertiary oil recovery and the effective viscosity of polymer solutions, two sets of sand packed flood tests were carried out by injecting polymer solutions of different viscosities. The additional oil recoveries by polymer flooding were $19.21 \%$ and $21.00 \%$ OOIP for $2000 \mathrm{ppm}$ and 2500 ppm PHPA solutions, respectively.

\section{Conflict of Interests}

The authors declare that there is no conflict of interests regarding the publication of this paper.

\section{Acknowledgments}

The authors gratefully acknowledge the financial assistance provided by University Grant Commission [F. no. 37-203/ 2009 (SR)], New Delhi, to the Department of Petroleum Engineering, Indian School of Mines, Dhanbad, India. Thanks are also extended to all individuals associated with the project.

\section{References}

[1] P. L. J. Zitha, K. G. S. van Os, and K. F. J. Denys, "Adsorption of linear flexible polymers during laminar flow through porous media: effect of concentration," in Proceedings of the SPE/DOE Improved Oil Recovery Symposium, pp. 19-22, Tulsa, Okla, USA, April 1998, SPE paper no. 39675.

[2] O. L. Kouznetsov, E. M. Simkin, G. V. Chilingar, and S. A. Katz, "Improved oil recovery by application of vibro-energy to waterflooded sandstones," Journal of Petroleum Science and Engineering, vol. 19, no. 3-4, pp. 191-200, 1998.

[3] A. L. Ogunberu and K. Asghari, "Water permeability reduction under flow-induced polymer adsorption," in Proceedings of the 5th Canadian International Conference, Alberta, Canada, June 2004, paper no. 2004-236.

[4] P. D. Moffitt, "Long-term production results of polymer treatments in producing wells in western Kansas," Journal of Petroleum Technology, vol. 45, no. 4, pp. 356-362, 1993.

[5] A. Zaitoun and N. Kohler, "Two phase flow through porous Media: effect of an Adsorbed Polymer Layer," in Proceedings of the SPE Annual Technical Conference and Exhibition, Huston, Tex, USA, October 1998, SPE paper no. 18085.

[6] M. A. Mohammed, "Investigation of polymer adsorption on rock surface of high saline reservoirs," in Proceedings of the Saudi Arabia Section Technical Symposium (SPE '08), Alkhobar, Saudi Arebia, May 2008, SPE paper no. 120807.

[7] H. T. Dovan and R. D. Hutchins, "New polymer technology for water control in gas wells," SPE Production and Facilities, vol. 9, no. 4, pp. 280-286, 1994.

[8] G. J. Hirasaki and G. A. Pope, "Analysis of factors influencing mobility and adsorption in the flow of polymer solution through porous media," Society of Petroleum Engineering Journal, vol. 14, no. 4, pp. 337-346, 1974. 
[9] C. G. Zheng, B. L. Gall, H. W. Gao, A. E. Miller, and R. S. Bryant, "Effects of polymer adsorption and flow behavior on two-phase flow in porous media," SPE Reservoir Evaluation \& Engineering, vol. 3, no. 3, pp. 216-223, 2000.

[10] A. Zaitoun and H. Bertin, "Two-phase flow property modification by polymer adsorption," in Proceedings of the SPE Improved Oil Recovery Symposium, Tulsa, Okla, USA, 1998, SPE Paper no. 39631.

[11] B. C. A. Grattoni, P. F. Luckham, X. D. Jing, L. Norman, and R. W. Zimmerman, "Polymers as relative permeability modifiers: adsorption and the dynamic formation of thick polyacrylamide layers," Journal of Petroleum Science and Engineering, vol. 45, no. 3-4, pp. 233-245, 2004.

[12] N. Lai, X. Qin, Z. Ye, C. Li, K. Chen, and Y. Zhang, "The study on permeability reduction performance of a hyperbranched polymer in high permeability porous medium," Journal of Petroleum Science and Engineering, vol. 112, pp. 198-205, 2013.

[13] A. R. Al-Hashmi and P. F. Luckham, "Characterization of the adsorption of high molecular weight non-ionic and cationic polyacrylamide on glass from aqueous solutions using modified atomic force microscopy," Colloids and Surfaces A, vol. 358, no. 1-3, pp. 142-148, 2010.

[14] H. H. Al-Sharji, C. A. Grattoni, R. A. Dawe, and R. W. Zimmerman, "Disproportionate permeability reduction due to polymer adsorptrion-entanglement," in SPE European Formation Damage Conference, The Hague, Netherlands, 2001, SPE Paper no. 68972.

[15] S. Liu, D. L. Zhang, W. Yan, M. Puerto, G. J. Hirasaki, and C. A. Miller, "Favorable attributes alkaline-surfactant-polymer flooding," Society of Petroleum Engineering Journal, vol. 13, no. 1, pp. 5-16, 2008.

[16] Y. Masuda, K.-C. Tang, M. Miyazawa, and S. Tanaka, "1D simulation of polymer flooding including the viscoelastic effect of polymer solution," SPE Reservoir Engineering, vol. 7, no. 2, pp. 247-252, 1992.

[17] A. Bera, T. Kumar, K. Ojha, and A. Mandal, "Adsorption of surfactants on sand surface in enhanced oil recovery: isotherms, kinetics and thermodynamic studies," Applied Surface Science, vol. 284, pp. 87-99, 2013.

[18] W. Lv, B. Bazin, D. Ma, Q. Liu, D. Han, and K. Wu, "Static and dynamic adsorption of anionic and amphoteric surfactants with and without the presence of alkali," Journal of Petroleum Science and Engineering, vol. 77, no. 2, pp. 209-218, 2011.

[19] M. Safian-Boldani, M. P. Shahri, M. Zargartalebi, and M. Arabloo, "New surfactant extracted from zizyphus spina christi for enhanced oil recovery: experimental determination of static adsorption isotherm," Journal of the Japan Petroleum Institute, vol. 56, no. 3, pp. 142-149, 2013.

[20] M. A. Ahmadi and S. R. Shadizadeh, "Experimental investigation of adsorption of a new nonionic surfactant on carbonate minerals," Fuel, vol. 104, no. 2, pp. 462-467, 2013.

[21] A. Samanta, K. Ojha, A. Sarkar, and A. Mandal, "Mobility control and enhanced oil recovery using partially hydrolyzed polyacrylamide (PHPA)," International Journal of Oil, Gas Coal Technology, vol. 6, no. 3, pp. 245-258, 2013.

[22] D. D. Sparlin, "Evaluation of polyacrylamides for reducing water production (includes associated papers 6561 and 6562)," Journal of Petroleum Technology, vol. 28, no. 5, pp. 906-914, 1976.

[23] Y. Cohen and A. B. Metzner, "Adsorption effects in the flow of polymer solutions through capillaries," Macromolecules, vol. 15, no. 5, pp. 1425-1429, 1982.
[24] N. Tekin, A. Dinçer, Ö. Demirbaş, and M. Alkan, "Adsorption of cationic polyacrylamide (C-PAM) on expanded perlite," Applied Clay Science, vol. 50, no. 1, pp. 125-129, 2010.

[25] N. Tekin, Ö. Demirbaş, and M. Alkan, "Adsorption of cationic polyacrylamide onto kaolinite," Microporous and Mesoporous Materials, vol. 85, no. 3, pp. 340-350, 2005.

[26] B. K. Maitin, "Performance analysis of several polyacrylamide floods in the North German oil fields," in Proceedings of the SPE/DOE Enhance Oil Recovery Symposium, Tulsa, Okla, USA, April 1992, SPE paper no. 24118. 

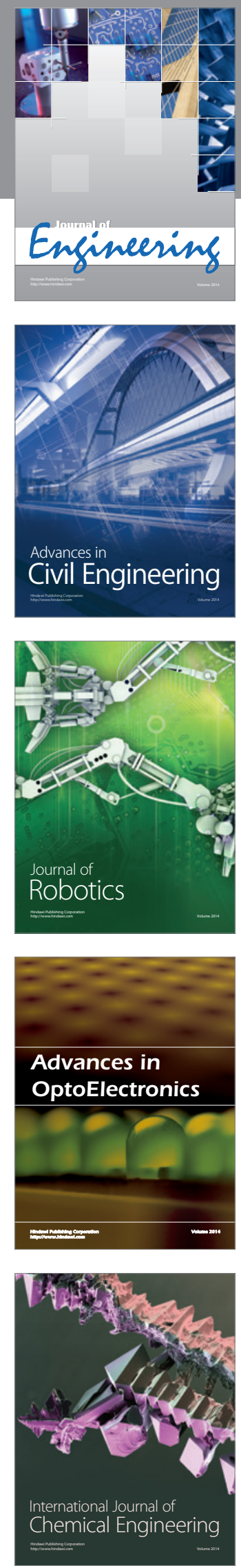

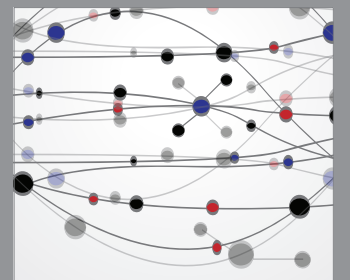

The Scientific World Journal
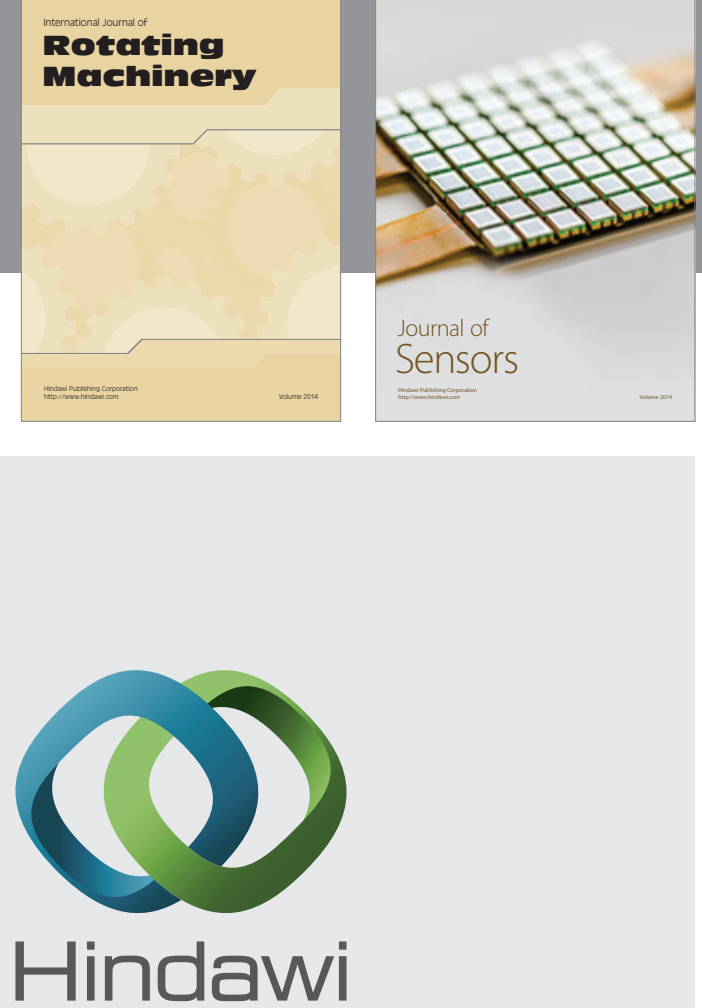

Submit your manuscripts at http://www.hindawi.com
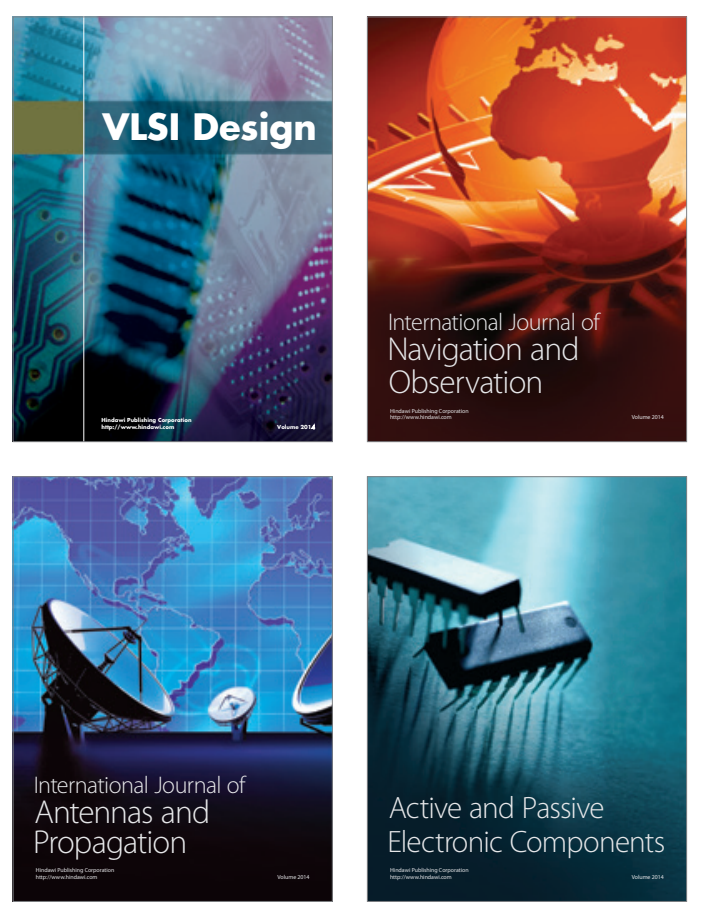
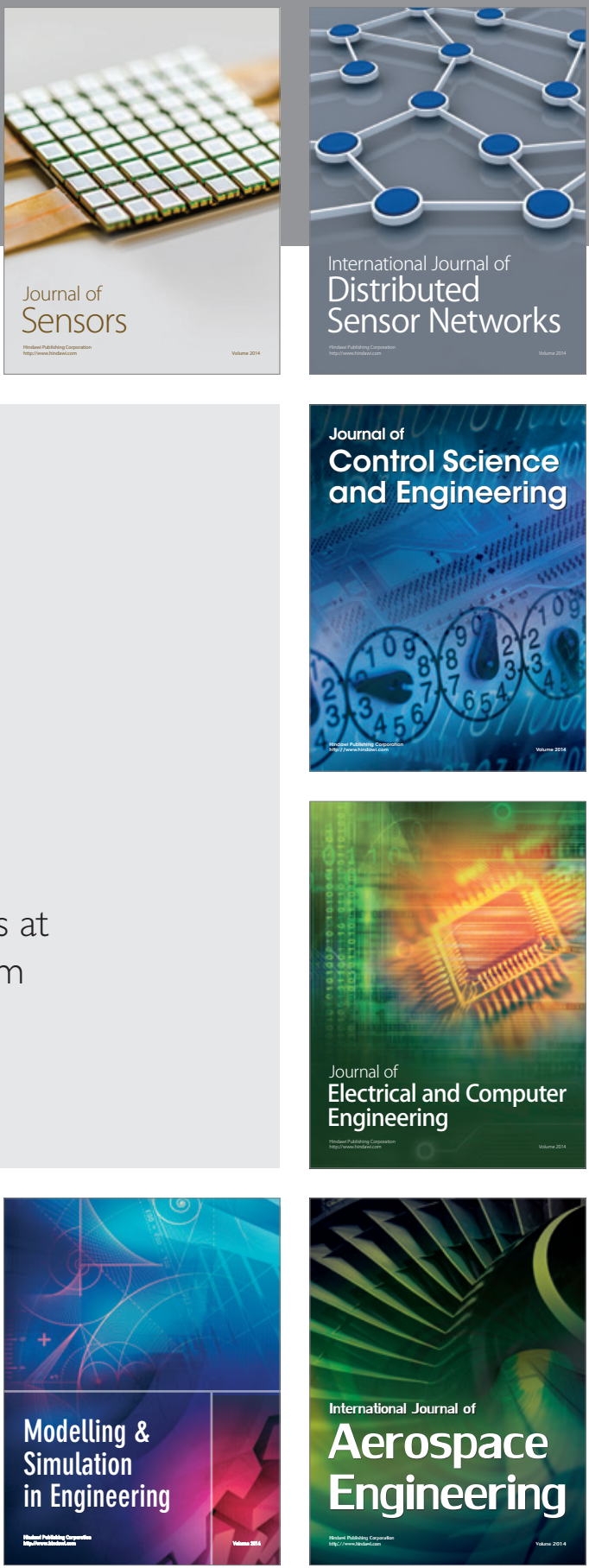

Journal of

Control Science

and Engineering
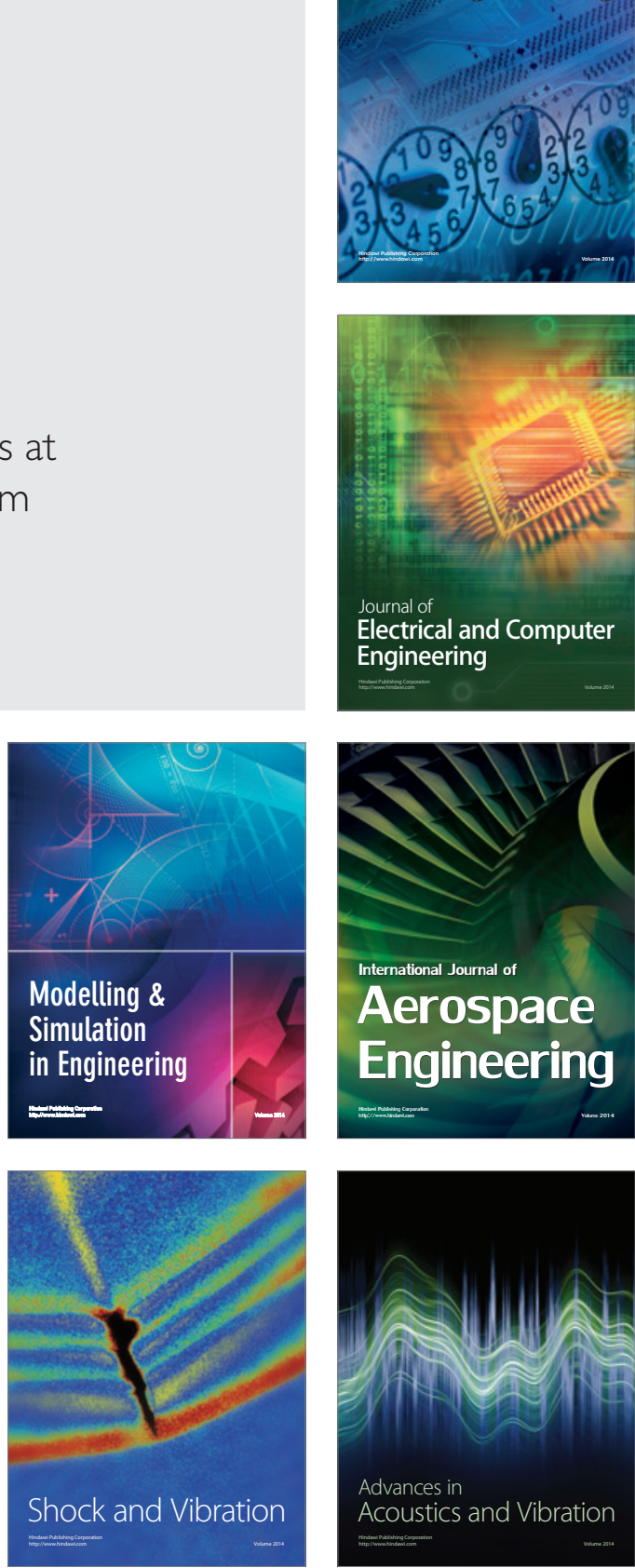